\title{
Bone Sarcoma
}

National Cancer Institute

\section{Source}

National Cancer Institute. Bone Sarcoma. NCI Thesaurus. Code C9312.

A sarcoma that arises from the bone. Representative examples are osteosarcoma and chondrosarcoma. 